\title{
Charge Separation and Exciton Dynamics at Polymer/ZnO Interface from First-Principles Simulations
}

\author{
Guangfen $\mathrm{Wu}, \mathrm{Zi} \mathrm{Li}, \mathrm{Xu}$ Zhang, and Gang Lu* \\ Department of Physics and Astronomy, California State University Northridge, Northridge, California 91330-8268, United States \\ Supporting Information
}

ABSTRACT: Charge separation and exciton dynamics play a crucial role in determining the performance of excitonic photovoltaics. Using time-dependent density functional theory with a range-separated exchange-correlation functional as well as nonadiabatic ab initio molecular dynamics, we have studied the formation and dynamics of charge-transfer (CT) excitons at polymer/ZnO interface. The interfacial atomic structure, exciton density of states and conversions between exciton species are examined from first-principles. The exciton dynamics exhibits both adiabatic and nonadiabatic characters. While the adiabatic transitions are facilitated by $\mathrm{C}=\mathrm{C}$ vibrations along the polymer ( $\mathrm{P} 3 \mathrm{HT}$ ) backbone, the nonadiabatic transitions are realized by exciton hopping between the excited states. We find that the localized $\mathrm{ZnO}$ surface states lead to localized low-energy CT states and poor charge separation. In contrast, the surface states of crystalline $\mathrm{C}_{60}$ are indistinguishable from the bulk states,

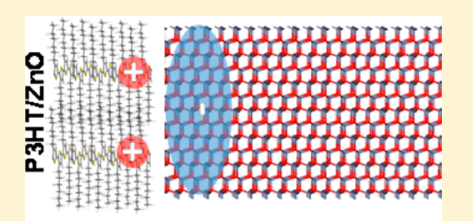
resulting in delocalized CT states and efficient charge separation in polymer/fullerene (P3HT/

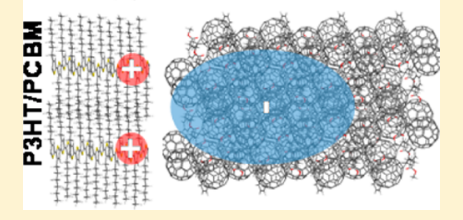
PCBM) heterojunctions. The hot CT states are found to cool down in an ultrafast time scale and may not play a major role in charge separation of $\mathrm{P} 3 \mathrm{HT} / \mathrm{ZnO}$. Finally we suggest that the dimensions of nanostructured acceptors can be tuned to obtain both efficient charge separation and high open circuit voltages.

SECTION: Energy Conversion and Storage; Energy and Charge Transport

$\mathrm{O}$ rganic/inorganic hybrid solar cells based on organic polymers and inorganic nanocrystallites have attracted great interest in the development of low-cost, scalable and robust photovoltaic technologies. ${ }^{1-7}$ Combining the strengths of $p$-type organic materials such as high absorption coefficients and flexibility with the advantages of $n$-type inorganic semiconductors such as high electron mobilities, superior stability and ability to form various nanostructures, the hybrid solar cells can achieve unique functionalities that cannot be obtained for each component alone. However, despite their tantalizing potentials, the power conversion efficiency (PCE) of the hybrid solar cells remains rather low comparing to allorganic photovoltaics (OPVs). For example, the PCE of a prototypical hybrid solar cell, poly(3-hexylthiophene) (P3HT)/ $\mathrm{ZnO}$ is less than $\sim 2 \%,{ }^{2,3,6,7}$ while its all-organic counterpart with phenyl-C61-butyric acid methyl ester (PCBM) replacing $\mathrm{ZnO}$ can reach $6 \%{ }^{8}$ Similarly, the internal quantum efficiency of $\mathrm{P} 3 \mathrm{HT} / \mathrm{ZnO}$ is about $30 \%$, ${ }^{9}$ comparing to nearly $100 \%$ of P3HT/PCBM. ${ }^{10}$

Although the factors controlling PCE are not yet fully understood, it is widely accepted that charge separation at donor/acceptor (D/A) interfaces is the key. More specifically, the charge transfer (CT) excitons-intermediates between the donor excitons and separated charge carriers-play crucial roles in the photovoltaic performance, as both photocurrent and open circuit voltage $\left(V_{o c}\right)$ depend on them. ${ }^{11-13}$ Although presently there is no clear distinction between delocalized CT states and charge-separated states, we take the view here that the charge-separated states result from high-energy delocalized
CT states, ${ }^{11}$ whereas the low-energy localized CT states are loss channels for charge generation. There is evidence that charge separation may be enhanced by the generation of hot CT states and/or the existence of ordered domains on either side of the heterojunctions, enabling charge delocalization. ${ }^{14-18}$ Indeed, intense recent research has surged upon the role of hot CT excitons and their delocalization for charge separation in OPVs. ${ }^{14-22}$ In contrast, little attention has been paid to these aspects in the hybrid solar cells. This is unfortunate because the organic/inorganic heterojunctions are actually excellent systems to elucidate the role of hot CT excitons and charge delocalization. It has been shown that CT excitons are important in charge separation of $\mathrm{P} 3 \mathrm{HT} / \mathrm{ZnO}$ hybrid solar cells. $^{23}$ Moreover, unlike their organic counterparts, the inorganic acceptors are crystalline, thus providing "ideal” D/A interfaces for charge separation. Finally, a comparative study between OPVs and hybrid solar cells could offer insights that cannot be gleaned from the study of OPVs alone.

In this work, we investigate the formation and dynamics of $\mathrm{CT}$ excitons at $\mathrm{P} 3 \mathrm{HT} / \mathrm{ZnO}$ interface from first principles. The $\mathrm{P} 3 \mathrm{HT} / \mathrm{ZnO}$ interface is modeled by an ideal bilayer structure without considering extrinsic factors such as the presence of ligands, interfacial modifiers and surface defects. ${ }^{7}$ These extrinsic factors including space-charge layers and trap states could certainly influence charge separation at the interface, but

Received: May 16, 2014

Accepted: July 22, 2014

Published: July 22, 2014 
(a)

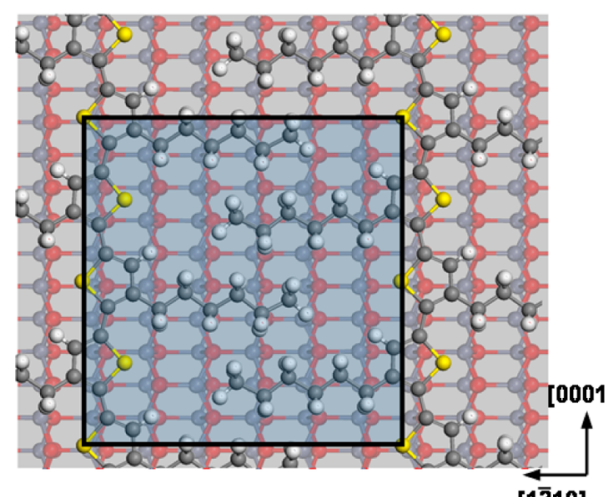

(d) (b)

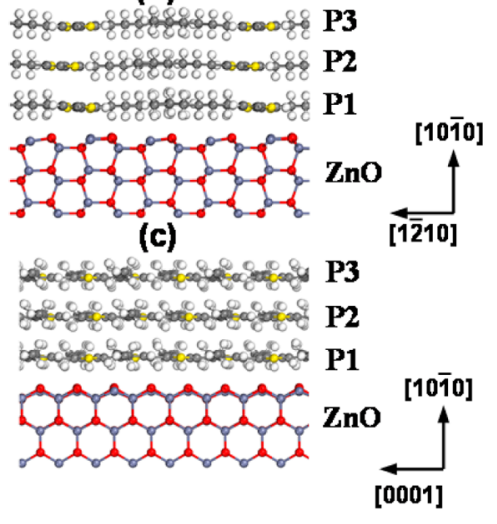

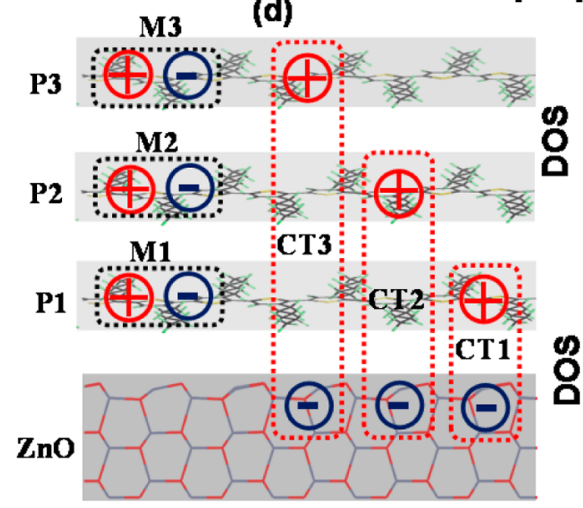

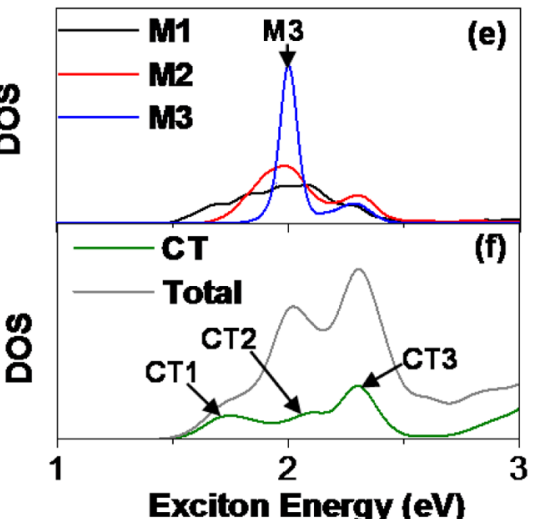

Figure 1. (a) Top and (b,c) side view of $\mathrm{P} 3 \mathrm{HT} / \mathrm{ZnO}$ interfacial atomic structure. (d) Schematics of excitons studied including intramolecular (M1, M2, M3) and charge transfer (CT1, CT2, CT3) excitons. (e,f) The exciton DOS of P3HT/ZnO with the ground state energy set to 0 . The blue, red, gray, yellow, and white spheres in panels a-c represent $\mathrm{Zn}, \mathrm{O}, \mathrm{C}, \mathrm{S}$, and $\mathrm{H}$ atoms, respectively.

are too complicated to be included in the present paper. Instead, the focus of this work is to understand intrinsic mechanisms underlying the inefficient charge separation at $\mathrm{ZnO} / \mathrm{P} 3 \mathrm{HT}$ interface, which is expected to be general among hybrid solar cells. The atomic structure of the interface and the density of states (DOS) of interfacial excitons are calculated from density functional theory (DFT). We reveal that localized surface states of a perfect and clean crystalline $\mathrm{ZnO}$ are the origin of localized $\mathrm{CT}$ states and poor charge separation at $\mathrm{P} 3 \mathrm{HT} / \mathrm{ZnO}$ interfaces. In contrast, the surface states of crystalline $\mathrm{C}_{60}$ are indistinguishable from the bulk states, resulting in delocalized $\mathrm{CT}$ states and efficient charge separation at P3HT/PCBM heterojunctions. We find that hot CT states can relax to the lowest CT state in an ultrafast time scale in $\mathrm{P} 3 \mathrm{HT} / \mathrm{ZnO}$, and hence are unlikely to play a major role in charge separation. Finally we show that the dimensions of nanostructured acceptors can influence both charge separation and $V_{o c}$ and optimized dimensions can be tuned to strike a balance between them. All computational details are provided in the Computational Methods section.

Interfacial Structure. We model the charge separation and exciton dynamics across a bilayer $\mathrm{P} 3 \mathrm{HT} / \mathrm{ZnO}$ interface using a slab model in which three P3HT layers are placed epitaxially on

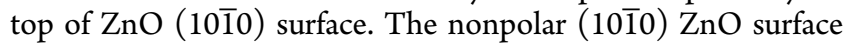
is chosen because it is the most energetically stable $\mathrm{ZnO}$ termination and has no dangling bonds or surface reconstruction. ${ }^{24}$ The lattice constants of wurtzite- $\mathrm{ZnO}$ are calculated as $a=3.24 \AA$ and $c=5.23 \AA$, close to the experimental values of a $=3.25 \AA$ and $\mathrm{c}=5.21 \AA^{25}$ The $(10 \overline{1} 0) \mathrm{ZnO}$ surface is modeled by a three double-layer slab with a $5 \times 3$ two-dimensional unit cell. Each P3HT chain with four thiophene rings is oriented along the surface trench in $[1 \overline{2} 10]$ direction between two surface dimer rows (Figures $1 \mathrm{a}-\mathrm{c}$ ), which has been shown to be energetically most stable. ${ }^{26-28}$ Since the monomer length of P3HT is $7.8 \AA \AA^{29}$ the lattice mismatch between P3HT and $\mathrm{ZnO}$ surface is exceedingly small $(\sim 0.1 \%)$. The three P3HT chains are labeled as P1, P2, and P3 in the order of their proximity to $\mathrm{ZnO}$ (Figures $1 \mathrm{~b}-\mathrm{d}$ ). The interplanar spacing between the $\mathrm{P} 1$ backbone and $\mathrm{ZnO}$ surface is $2.64 \AA$, close to the theoretical value $(2.6 \AA)$ from MP2 calculations; ${ }^{26}$ the average interchain distance between the three P3HTs is $\sim 3.6 \AA$, similar to the experimental value of $3.8 \AA .^{30}$ The thiophene rings in the adjacent P3HT chains have a relative shift of $\sim 1 / 2$ ring diameter.

Exciton Density of States. As the energy of excitons determines their dynamics, we calculate exciton density of states from ab initio Molecular Dynamics (AIMD). Three types of excitons are examined here: intramolecular, intermolecular, and CT excitons (Figure 1d). For the intramolecular (intermolecular) excitons, the quasi-electrons and quasi-holes are located on the same (different) P3HT molecule(s). The lowest-energy intramolecular exciton on P1, P2, and P3 is labeled as M1, $\mathrm{M} 2$, and $\mathrm{M} 3$, respectively. For CT excitons, the electrons reside at $\mathrm{ZnO}$ whereas the holes spread onto different P3HT chains. The lowest-energy CT exciton with the hole on P1, P2, and P3 is named as CT1, CT2, and CT3, respectively. The CT excitons at the $\mathrm{P} 3 \mathrm{HT} / \mathrm{ZnO}$ interface has been observed experimentally. ${ }^{23}$ Figure 1e,f displays the exciton DOS averaged over 1000 AIMD snapshots at $300 \mathrm{~K}$. The thermally averaged energies of $\mathrm{M} 1, \mathrm{M} 2$, and M3 are about the same $(\sim 2.0 \mathrm{eV})$, and the 
averaged energy of the lowest intermolecular exciton is $\sim 2.3 \mathrm{eV}$ with the electron and hole lying on adjacent $\mathrm{P} 3 \mathrm{HT}$ molecules. The average energy of CT1, CT 2 and CT 3 is $1.7,2.0$, and 2.3 $\mathrm{eV}$, respectively and the lowest excitation at the $\mathrm{P} 3 \mathrm{HT} / \mathrm{ZnO}$ heterojunction is CT1. The energy range of these relevant excitons $(1.7-2.3 \mathrm{eV})$ is in a good agreement with that from the photoinduced absorption spectroscopy measurement (1.9$2.5 \mathrm{eV}) .^{31}$

Interfacial Exciton Dynamics and Formation of CT States. Next, we examine exciton dynamics and the formation of CT states at the $\mathrm{P} 3 \mathrm{HT} / \mathrm{ZnO}$ interface. In Figures $2 \mathrm{a}$, b we display the time
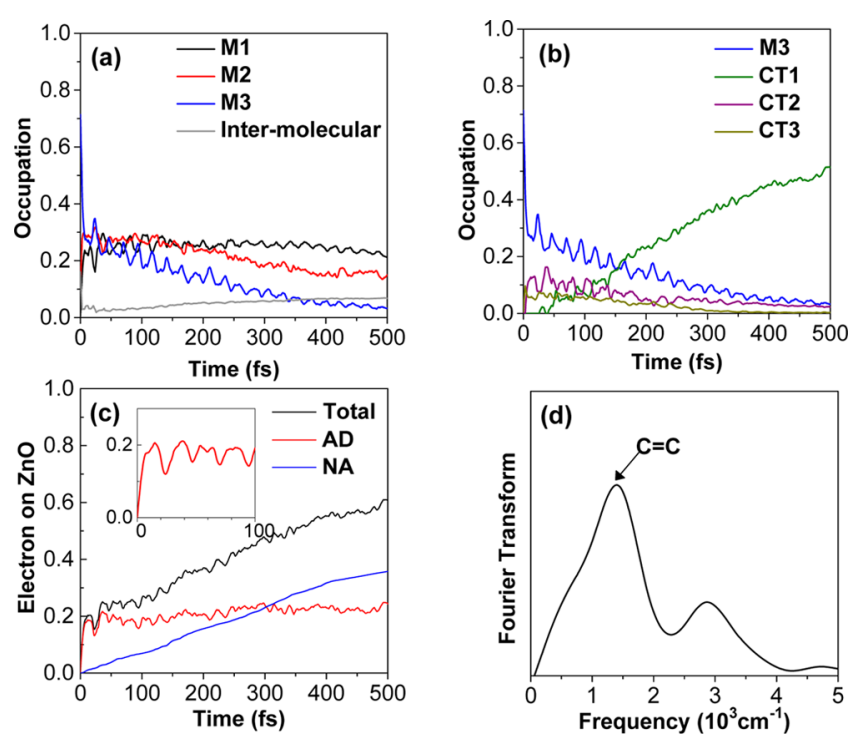

Figure 2. Exciton evolution with $\mathrm{M} 3$ as the initial state: (a) evolution dynamics of excitons on P3HT; (b) evolution dynamics of CT excitons; (c) electron dynamics on $\mathrm{ZnO}$. The adiabatic ( $\mathrm{AD}$ ), nonadiabatic (NA) and the sum of the two contributions (Total) are shown in different colors. The inset in panel $\mathrm{c}$ is the blown-up view in 100 fs. (d) The Fourier transform of the red curve in panel c.

evolution of a singlet exciton whose initial state is comprised primarily (80\%) of M3. This is the most distant intramolecular exciton afforded by the present simulations. The exciton converts rapidly to $\mathrm{M} 1$ and $\mathrm{M} 2$ in a time scale of $\sim 20 \mathrm{fs}$ as shown in Figure 2a. Driven by the energy resonance, M1 and M2 acquire a similar occupation fraction $(\sim 25 \%)$ as M3 at 100 fs. The conversion of M3 to CT states is shown in Figure $2 b$. The "hot" CT excitons (CT2 and CT3) are formed first, and subsequently relax to the lowest CT state (CT1). Confined by the size of the computational model, CT3 is the most energetic CT state $(0.6 \mathrm{eV}$ higher than CT1) examined in this work. The formation of the CT states constitutes both adiabatic and nonadiabatic contributions as shown in Figure 2c. The former is the result of quantum coherence driven by energy resonance between the relevant exciton states, whereas the latter is accomplished by exciton hopping between these states. The adiabatic contribution increases rapidly in the first few femtoseconds and then oscillates with a periodicity $(\sim 20 \mathrm{fs})$ that matches the frequency of $\mathrm{C}=\mathrm{C}$ vibration at $\sim 1450 \mathrm{~cm}^{-1}$. Therefore, the stretching mode of $\mathrm{C}=\mathrm{C}$ bonds along P3HT backbone facilitates the energy resonance between the exciton states, and the adiabatic transitions. This result is consistent with a recent experimental and computational finding ${ }^{32}$ that the $\mathrm{C}=\mathrm{C}$ vibrational mode with a periodicity of $23 \mathrm{fs}$ plays an important role in coherent charge transfer at $\mathrm{P} 3 \mathrm{HT} / \mathrm{PCBM}$ interfaces and other carbon-based organic systems. ${ }^{33,34}$ In contrast, the nonadiabatic contribution increases much slower but overcomes the adiabatic contribution after $300 \mathrm{fs}$. The dynamics of other exciton states has also been studied and is summarized in Figure 3. We find that the intramolecular

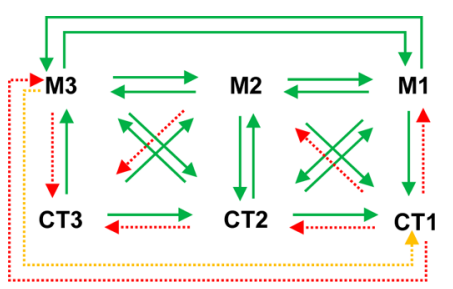

Figure 3. Diagram of possible exciton conversions. Green line: transitions observed; yellow line: energetic downhill but with weak wave function coupling; red line: energetic uphill process.

excitons can readily convert to each other thanks to their energy resonance. While the higher energy CT states can transit to the lower ones, the reverse is not observed in the simulations. M1 and M2 can convert to CT1 and CT2, but not to CT3. In general, the allowed transitions are energetic downhill while the forbidden ones are energetic uphill. There is a negligible transition from M3 to CT1 due to their poor coupling even though the process is energetic downhill. M3 and M2 can convert to hot CT states such as CT2, which subsequently cool down to CT1. Importantly, only the intramolecular excitons that are adjacent to the interface exhibit significant overlap with the CT states and hence convert directly to them. This observation is consistent with the experimental finding that the exciton dissociation efficiency drops by 1 order of magnitude as it moves from the first donor layer to the second across the interface. ${ }^{35}$ The hot CT states can also convert to the intramolecular excitons provided that the requirements of the energy and the wave function overlap are met.

Charge Localization and Surface States of the Acceptors. It is generally accepted that charge delocalization at the D/A interface is key to efficient charge separation. ${ }^{15,18,36}$ Ordered domains of fullerenes in OPVs ${ }^{18}$ and crystalline acceptor ${ }^{23}$ in hybrid solar cells are believed to accommodate such delocalized states for efficient charge separation. However, it is puzzling why charge separation in hybrid solar cells such as $\mathrm{P} 3 \mathrm{HT} / \mathrm{ZnO}$ is significantly poorer than that in OPVs such as P3HT/ PCBM? This is a poignant question for two reasons: (1) the crystallinity of $\mathrm{ZnO}$ is as high as that of $\mathrm{PCMB}$ at the interface, if not higher. (2) The exciton binding energy at P3HT/PCBM interface should be higher than that at $\mathrm{P} 3 \mathrm{HT} / \mathrm{ZnO}$ interface due to the higher dielectric constant of $\mathrm{ZnO}$. To answer this question, we examine the delocalization of CT states at the $\mathrm{P} 3 \mathrm{HT} / \mathrm{ZnO}$ interface. The charge density distribution of CT1, $\mathrm{CT} 2$, and CT3 is shown in Figures $4 \mathrm{a}-\mathrm{c}$, respectively. We immediately note that these CT states are localized at the interface. In particular, the electron is confined at the $\mathrm{ZnO}$ surface layers, while the hole is located at the P1, P2, and P3, respectively. It is remarkable that even CT3 is localized despite its higher energy. In Figure 4d, we show the charge density of a hot CT state whose hole resides at P1. Since its energy is higher than CT3, its charge density is slightly more delocalized than CT3 with a larger electron-hole distance. The overall localized nature of $\mathrm{CT}$ states, underlying the poor charge separation at the $\mathrm{P} 3 \mathrm{HT} / \mathrm{ZnO}$ interface, is in a sharp contrast with delocalized 


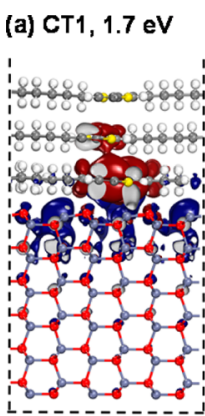

(b) $\mathrm{CT} 2,2.0 \mathrm{eV}$

(c) $\mathrm{CT} 3,2.3 \mathrm{eV}$
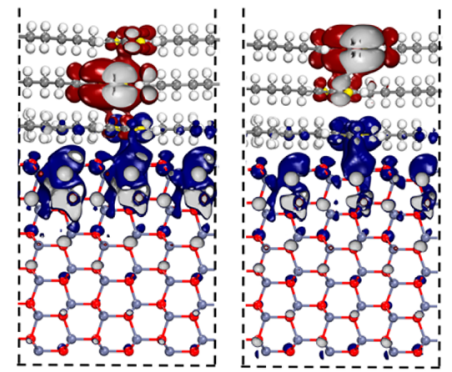

(d) Hot CT, $3.4 \mathrm{eV}$

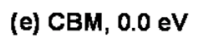

(f) $\mathrm{CBM}+19,2.0 \mathrm{eV}$

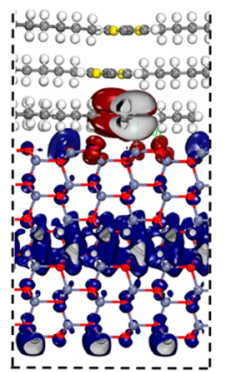

(e) CBM, $0.0 \mathrm{eV}$

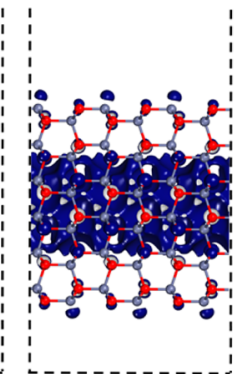

Figure 4. Charge density of CT1 (a), СT2 (b), СT3 (c), and the hot CT state with the hole on P1 (d). The red and blue color represents the charge density of the hole and the electron, respectively. The isosurface value is $5 \times 10^{-4} \mathrm{e} / \AA^{3}$. Panels e and f display the ground state band-decomposed charge density for the CBM and CBM+19. The isosurface value is $10^{-3} \mathrm{e} / \AA^{3}$. The white, gray, yellow, red, and blue spheres denote $\mathrm{H}, \mathrm{C}, \mathrm{S}, \mathrm{O}$, and $\mathrm{Zn}$ atoms, respectively.
CT states in P3HT/PCBM with ordered fullerene acceptors. ${ }^{16}$ An ensuring question is why the low-energy CT states are localized in $\mathrm{ZnO}$ but delocalized in crystalline PCBM? To answer this question, we calculate the band-decomposed ground state charge density for both $\mathrm{ZnO}$ and crystalline $\mathrm{C}_{60}$ surfaces. More specifically, the charge density contributions from the conduction-band-minimum (CBM) and CBM+19 of $\mathrm{ZnO}(10 \overline{1} 0)$ surface are determined using a slab model as shown in Figures 4e,f. Similarly, the charge density contributions from the lowest-unoccupied-molecular-orbital (LUMO), $\mathrm{LUMO}+1$, and LUMO+9 of the crystalline $\mathrm{C}_{60}$ surface are also displayed in Figures $5 \mathrm{a}-\mathrm{c}$ for comparison. The $\mathrm{C}_{60}$ molecules are arranged in a simple cubic lattice with 10 molecular layers between the two surfaces. The side-chains of PCMB are removed to render the calculations feasible. We find that the conduction band states of $\mathrm{ZnO}(10 \overline{1} 0)$ from $\mathrm{CBM}$ to $\mathrm{CBM}+18$ are all localized surface states, exemplified by CBM in Figure 4e. Some of the higher energy CB states (such as CBM+19) reside in the center of the slab model as shown in Figure $4 \mathrm{f}$. Since the surface CB states are likely to be occupied by the CT electrons, the localized surface states would lead to the localized $\mathrm{CT}$ states, whereas the higher energy $\mathrm{CB}$ states contribute to the hot CT states as shown in Figure 4d. The large energy difference $(\sim 1.7 \mathrm{eV})$ between $\mathrm{CT} 1$ and the hot CT state stems from the substantial energy difference $(\sim 2.0 \mathrm{eV})$ between the $\mathrm{CBM}$ and $\mathrm{CBM}+19$. On the contrary, the charge density of the unoccupied states in the crystalline $\mathrm{C}_{60}$ surface exhibits a delocalized character in general. For example, the LUMO charge density delocalizes to a length-scale of $4 \mathrm{~nm}\left(4 \mathrm{C}_{60}\right.$ molecules) underneath the each surface. Remarkably, this is the same length-scale as observed in PCBM-based OPVs for the

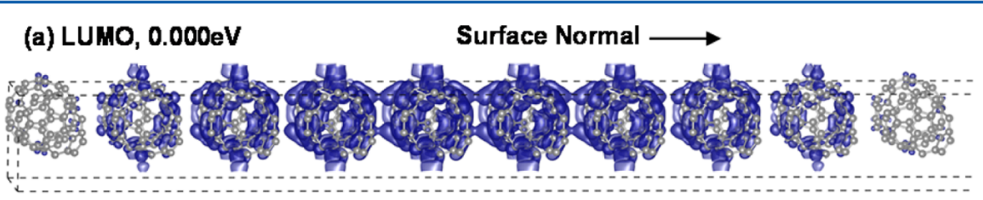

(b) LUMO+1, $0.005 \mathrm{eV}$

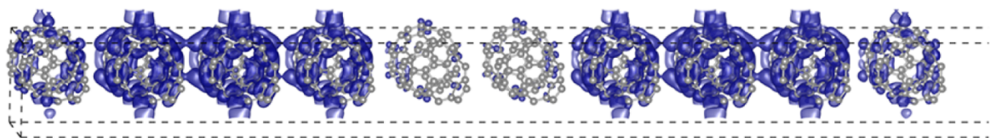

(c) LUMO+9, $0.10 \mathrm{eV}$
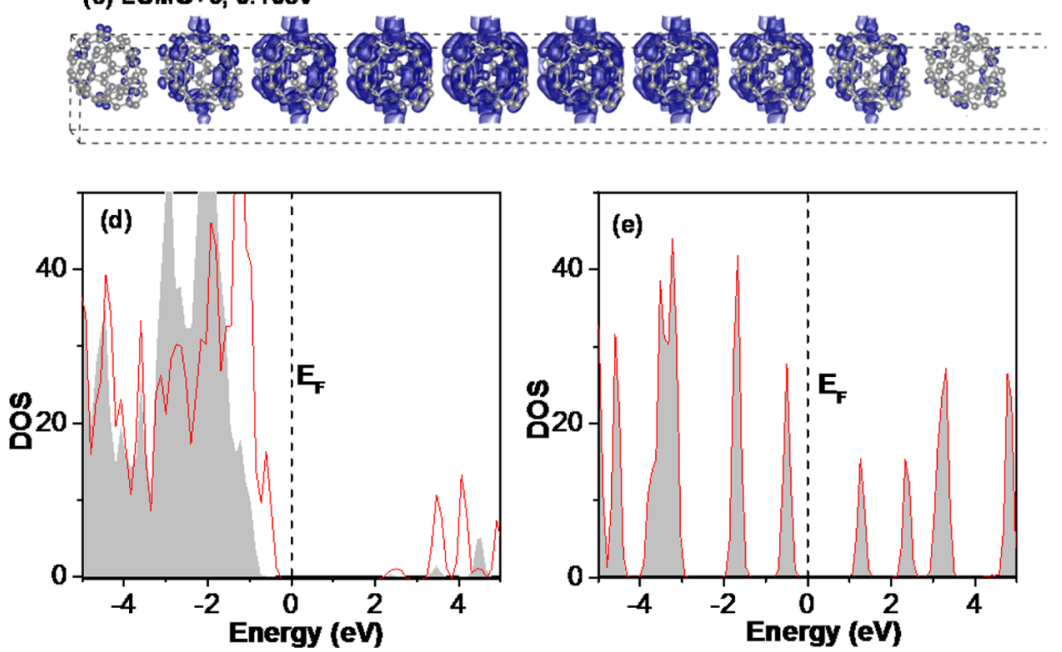

Figure 5. Band-decomposed charge density $(\mathrm{a}-\mathrm{c})$ and density of states for $\mathrm{C}_{60}$ surface in a simple cubic crystal structure (e) and $\mathrm{ZnO}(10 \overline{1} 0)$ surface (d). The gray spheres denote $\mathrm{C}$ atoms. The energy with respect to LUMO level is indicated in panels a-c. The Fermi level in panels $\mathrm{d}$ and e is set to zero. The red curve and gray area in panels $d$ and e represent the DOS contribution due to the surface and bulk, respectively. 
initial charge separation. ${ }^{18}$ Therefore, one can make a direct connection between charge separation and the existence of surface states at the acceptor. To elucidate this connection, we show the DOS contributions from the bulk states and the surface states for both crystalline $\mathrm{ZnO}$ and $\mathrm{C}_{60}$ surfaces in Figures 5 d,e. For $\mathrm{ZnO}$, the surface and bulk states have very different $\mathrm{CB}$ densities of states. In contrast, the surface states are almost identical to the bulk states in $\mathrm{C}_{60}$ as far as the DOS is concerned. In other words, the surface states in $\mathrm{ZnO}$ are prominent, whereas they are indistinguishable from the bulk states in $\mathrm{C}_{60}$. Since in general surface states are localized while bulk states are delocalized, the charge distribution at $\mathrm{ZnO}$ surface (and the P3HT/ZnO interface) is more localized than that at the PCBM surface (and the P3HT/PCBM interface). Because the bulk states are similar to each other, their energies are also close. The fact that there are many delocalized and nearly degenerate CT states at the P3HT/PCBM interface underscores its efficient charge separation. Finally, to verify that the localized CT states in $\mathrm{P} 3 \mathrm{HT} / \mathrm{ZnO}$ are indeed the consequence of the localized $\mathrm{ZnO}$ surface states and not due to the presence of $\mathrm{P} 3 \mathrm{HT}$, we increase the distance between P3HT and $\mathrm{ZnO}$ by $3.5 \AA$ relative to their equilibrium distance and calculate the charge density of CT1. We find that CT1 remains localized at the surface. Since the localized surface states are intrinsic to $\mathrm{ZnO}$, they should always be present independent of $\mathrm{ZnO}$ domain size and/or the presence of possible surface defects, and the low-energy $\mathrm{CT}$ states are always localized at the $\mathrm{P} 3 \mathrm{HT} / \mathrm{ZnO}$ interface. This is a fundamental problem for the hybrid solar cells, which may only be mitigated by eliminating the surface states. For example, one may introduce modifiers at the interface to make the inorganic surface mimic an organic one.

Hot CT States on Charge Separation. Recently, there have been significant interest and debate on the role of hot excitons in charge separation. ${ }^{14,21,22}$ On the one hand, Grancini et al. ${ }^{14}$ reported that the hot $\mathrm{CT}$ states dissociated before the internal conversions and led to a higher charge generation efficiency. In particular, the singlet excitons can convert into hot $\mathrm{CT}$ states and polarons in $20-50 \mathrm{fs}$ in $\mathrm{PCPDTBT} / \mathrm{PC}_{60} \mathrm{BM}$ heterojunctions. Similarly, Jailaubekov et al. $^{22}$ have examined the critical role of hot CT excitons in assisting charge separation. They showed that in phthalocyanine/fullerene heterojunctions, the hot CT states were formed in $100 \mathrm{fs}$ and cooled down on a time-scale of 1 ps. On the other hand, Vandewal et al. ${ }^{21}$ have concluded that for a wide range of OPVs, such as polymer/ fullerene, small-molecule $/ \mathrm{C}_{60}$ and polymer/polymer blends, hot CT states are not essential for charge separation owing to their ultrafast relaxation. Since the high-energy CT states are more delocalized than the low-energy ones, the efficiency of charge separation depends on the nature of the low-energy CT states; delocalized low-energy CT states lead to a high yield of charge carriers, whereas localized low-energy CT states produce poor charge separation. Our simulations suggest that the lowenergy CT states can relax to CT1 faster than the high-energy CT states, and both compete with the charge separation process. In particular, we find that in $\mathrm{P} 3 \mathrm{HT} / \mathrm{ZnO}$, the hot CT states can relax to CT1 and intramolecular excitons in an ultrafast time scale. As shown in Figure 6, the initial hot CT3 state converts to the intramolecular excitons in $20 \mathrm{fs}$ and in about 40 fs CT1 state starts to form. Similar results are observed for the initial hot CT2 state. Note that the formation time scale for the CT states in $\mathrm{P} 3 \mathrm{HT} / \mathrm{ZnO}$ is similar to that in OPVs, ${ }^{14,22}$ but the relaxation time scale is much faster than in

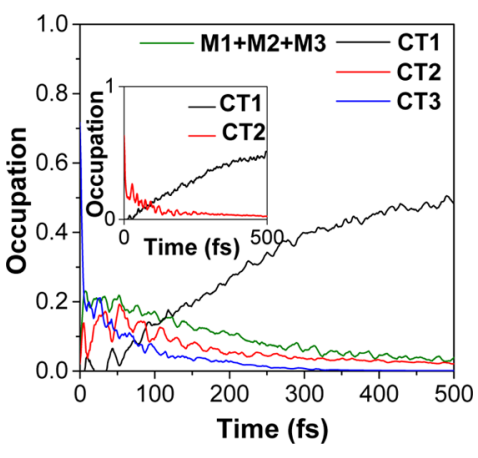

Figure 6. Exciton evolution with $\mathrm{CT} 3$ as the initial state; the inset is the evolution with CT2 as the initial state.

phthalocyanine/fullerene. Owing to the ultrafast relaxation and the localized nature of the low-energy CT states, we speculate that the hot CT states probably do not play an essential role in charge separation. However, future experiments and simulations are needed to ascertain the role of the hot excitons.

Quantum Size Effect on Charge Separation. In hybrid solar cells, inorganic nanostructures, such as nanosheets, nanoparticles, nanorods, and nanofibers are often used as acceptors. ${ }^{6,7}$ These nanostructures can improve the infiltration of the polymers into the free space of the oxides, increase the contact area, and enhance the interfacial interaction between the donor and the acceptor. Here we address another possible benefit of nanostructured inorganic acceptors, quantum size effect on charge separation, which has not been widely explored. To illustrate the idea, we employ a simple model with a P3HT molecule placed on top of $\mathrm{ZnO}(10 \overline{10})$ nanosheets (Figure 7).

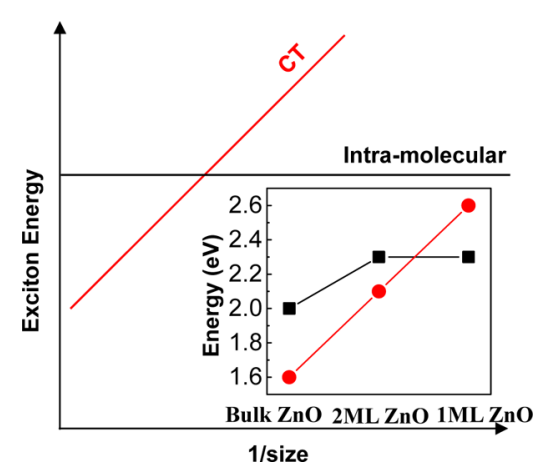

Figure 7. Exciton energy as a function of inverted size of $\mathrm{ZnO}$ nanosheets. Inset is the energy of the intramolecular and CT excitons as a function of the thickness of $\mathrm{ZnO}$ nanosheets. Squares and dots represent the intramolecular and $\mathrm{CT}$ excitons, respectively.

More specifically, we consider three cases: one-monolayer $\mathrm{ZnO}$, two-monolayer $\mathrm{ZnO}$, and "bulk" $\mathrm{ZnO}$ as acceptor. In the first two cases, the atomic positions and the lattice constants are fully relaxed and in the "bulk" case, three $\mathrm{ZnO}$ layers are used in the slab model with the atoms in the bottom layer fixed to their equilibrium bulk positions. We find that the energy of the CT1 state increases rapidly as the thickness of $\mathrm{ZnO}$ nanosheets decreases, while the energy of the intramolecular exciton is essentially unchanged between the first two cases. This is because reducing the thickness of the nanosheets increases its energy level separations, hence its excitation energies. The intramolecular exciton energy depends primarily on the donor material, thus is insensitive to the dimensions of the acceptor. In the model system, the conversion from the intramolecular 
exciton to the CT exciton is prerequisite for charge separation, and it is mediated by the energy resonance between the intramolecular and CT excitons. Therefore, for efficient charge separation, the nanostructure dimensions should be larger than some critical size so that the energy of the intramolecular exciton matches to that of the CT exciton. On the other hand, it has been shown both experimentally ${ }^{37}$ and computationally ${ }^{38}$ that the open circuit voltage $\left(V_{o c}\right)$ is proportional to the energy of CT excitons, hence a higher CT energy renders a higher $V_{\mathrm{oc}}$. These competing effects lead to an optimal dimension of the nanostructure in achieving both high charge separation efficiency (and high short-circuit current) and high $V_{\text {oc }}$. It would be interesting to confirm this idea experimentally whereby one tunes the dimensions of nanostructures in planar hybrid solar cells to achieve higher PCEs.

In summary, the interfacial exciton dynamics at $\mathrm{P} 3 \mathrm{HT} / \mathrm{ZnO}$ heterojunction is studied from first-principles. The interfacial atomic structure and exciton DOS are determined which provide the basis to understand the interfacial charge separation. The conversions among the intermolecular, intramolecular, and CT excitons are examined in detail. The excitons dynamics has both adiabatic and nonadiabatic contributions; the former is facilitated by the $\mathrm{C}=\mathrm{C}$ vibrations along the polymer backbone and dominates at the first few femtoseconds, whereas the latter takes over after 300 fs. Energetic driving force and wave function coupling are determinants of the exciton dynamics. We reveal that the localized $\mathrm{ZnO}$ surface states are the culprit behind inefficient charge separation, leading to the localized low-energy CT states. The localized surface states are intrinsic of the inorganic acceptors and independent of surface defects or the donors. In contrast, the surface states of the crystalline $\mathrm{C}_{60}$ are indistinguishable from the bulk states, yielding delocalized CT states and more efficient charge separation. We find that the hot CT states can cool down to CT1 in an ultrafast time scale. Finally we show that the dimensions of the nanostructured acceptors can influence both charge separation and $V_{\text {oc }}$.

\section{COMPUTATIONAL METHODS}

The exciton dynamics is simulated based on the timedependent density functional linear response theory (TDDFLRT) with a range-separated hybrid functional in conjunction with ab initio nonadiabatic molecular dynamics (NAMD) ${ }^{39,40}$ and modified fewest switches surface hopping method. ${ }^{41-43}$ The same approach has also been used to study exciton dynamics and diffusion in organic semiconductors, which compares very well to the experimental results. ${ }^{44,45}$ The similar methods have been used to study photoexcited charge transfer and recombination at quaterthiophene/ $\mathrm{ZnO}$ interface, ${ }^{46}$ charge transfer dynamics in dye-sensitized solar cells, ${ }^{4-49}$ and exciton dynamics in quantum dots, ${ }^{50}$ etc. The approximations involved in the methods include the fact that the electronic system is coupled to the nuclei through the nonadiabatic couplings only, while relaxation effects are captured by the Boltzmann factors introduced phenomenologically into the hopping probabilities. The excited state forces are not computed and the nuclear motion is only approximately determined. However, since our system is relatively large, the excited state forces are not expected to have a major influence on the nuclear motion. More technical details of these methods can be found in the Supporting Information and the references therein. In the TDDFT-LRT simulations, the range-separation parameter $\mu$ was chosen to be $0.2 \AA^{-1}$. Since this parameter depends on the length of $\pi$-conjugated polymer chains, ${ }^{51}$ we compute the optimally tuned $\mu$ value as a function of the number of thiophene rings $(N)$ in the polymer using the scheme proposed by Refaely-Abramson et al. ${ }^{52}$ By a linear fitting of $\mu$ as a function of $1 / N$, we can determine $\mu$ for $\mathrm{P} 3 \mathrm{HT}$ with an infinite length (Figure S1a). Using this $\mu$, we calculate the optical gap of $\mathrm{P} 3 \mathrm{HT}$ as $2.0 \mathrm{eV}$ and the quasiparticle transport gap of $\mathrm{ZnO}$ as $3.5 \mathrm{eV}$, both reproducing well the corresponding experimental value $\sim 2.0 \mathrm{eV}^{53}$ and $3.4 \mathrm{eV}^{25}$ respectively. Moreover, the computed energies of the relevant excitons $(1.7-2.3 \mathrm{eV})$ are similar to the experimental values (1.9-2.5 $\mathrm{eV}$ ) measured by the photoinduced absorption spectroscopy. ${ }^{31}$

Ground state DFT and ab initio Born-Openheimer MD (BOMD) simulations were performed using the Vienna $a b$ initio Simulation Package (VASP) ${ }^{54}$ with the projectoraugmented-wave potentials ${ }^{55}$ and generalized gradient approximations (GGA) for the exchange-correlation functional. The semiempirical DFT-D2 method $^{56}$ was used to account for the van der Waals (vdW) corrections with which the interfacial atomic structure was determined. The interface was modeled with three layers of $\mathrm{P} 3 \mathrm{HT}$, each with four thiophene rings, placed epitaxially on a $5 \times 3$ slab of $\mathrm{ZnO}(10 \overline{1} 0)$ atomic planes. As shown in the Supporting Information (Figure S1b), three double-layers of $\mathrm{ZnO}$ were sufficient to obtain converged exciton energies and used in the exciton dynamics simulations. The vacuum layer was of $15 \AA$ thick, which was large enough to decouple the interaction between the neighboring slabs. In the ground state structural relaxation, the atoms at the bottom double-layer of $\mathrm{ZnO}$ substrate were fixed to their equilibrium bulk positions, and the other atoms were allowed to relax. For the charge density calculations of the CT states, we used six double-layers of $\mathrm{ZnO}$ to provide an accurate description for the hot CT state (3.4 eV above CT1), which has a larger electronhole distance. The ground state charge density and DOS of $\mathrm{ZnO}$ and $\mathrm{C}_{60}$ surfaces were calculated using the hybrid functional HSE06. ${ }^{57} \mathrm{Six}$ double-layers of $\mathrm{ZnO}$ and ten layers of $\mathrm{C}_{60}$ were included in the surface slab models. The Gamma kpoint was sampled with a cutoff energy of $400 \mathrm{eV}$ in all calculations. We have also checked the results for the electronic structure and the exciton energies with $3 \times 3 \times 1$ and $5 \times 5 \times 1$ $\mathrm{k}$-points, and only minor energy differences were found. Based on the relaxed atomic structure, ab initio BOMD simulation was followed to raise the temperature to $300 \mathrm{~K}$ with repeated velocity scaling; the system was then kept at $300 \mathrm{~K}$ for 500 fs to reach the thermal equilibrium. Finally, a microcanonical BOMD production run was performed for $1000 \mathrm{fs}$ with a time-step of 1 fs. At each MD step, the excitation energies and the many-body wave functions are determined following Casida's formulation within TDDFT framework. To capture the stochastic nature of the coupled electron-ion dynamics, $900 \mathrm{ab}$ initio MD trajectories, each 100 fs long (some 500 fs long), were selected and averaged for the exciton dynamics from the 1000 fs long BOMD simulation. For each 100 fs trajectory, 100 surface hopping simulations were performed.

\section{ASSOCIATED CONTENT}

\section{S Supporting Information}

The choice of exchange-correlation functionals for the DFT calculations. The details of ab initio nonadiabatic molecular dynamics. Additional figures. This material is available free of charge via the Internet at http://pubs.acs.org. 


\section{AUTHOR INFORMATION}

\section{Corresponding Author}

*E-mail: ganglu@csun.edu.

Notes

The authors declare no competing financial interest.

\section{ACKNOWLEDGMENTS}

This work was supported by NSF-PREM Grant DMR-1205734 and the Department of Defense.

\section{REFERENCES}

(1) Huynh, W. U.; Dittmer, J. J.; Alivisatos, A. P. Hybrid NanorodPolymer Solar Cells. Science 2002, 295, 2425-2427.

(2) Oosterhout, S. D.; Wienk, M. M.; van Bavel, S. S.; Thiedmann, R.; Koster, L. J. A.; Gilot, J.; Loos, J.; Schmidt, V.; Janssen, R. A. J. The Effect of Three-Dimensional Morphology on the Efficiency of Hybrid Polymer Solar Cells. Nat. Mater. 2009, 8, 818-824.

(3) Hsu, J. W. P.; Lloyd, M. T. Organic/Inorganic Hybrids for Solar Energy Generation. MRS Bull. 2010, 35, 422-428.

(4) Chandrasekaran, J.; Nithyaprakash, D.; Ajjan, K. B.; Maruthamuthu, S.; Manoharan, D.; Kumar, S. Hybrid Solar Cell Based on Blending of Organic and Inorganic Materials-An Overview. Renew. Sust. Energy Rev. 2011, 15, 1228-1238.

(5) Wright, M.; Uddin, A. Organic-Inorganic Hybrid Solar Cells: A Comparative Review. Sol. Energy Mater. Sol. Cells 2012, 107, 87-111.

(6) Li, S.-S.; Chen, C.-W. Polymer-Metal-Oxide Hybrid Solar Cells. J. Mater. Chem. A 2013, 1, 10574-10591.

(7) Fan, X.; Zhang, M.; Wang, X.; Yang, F.; Meng, X. Recent Progress in Organic-Inorganic Hybrid Solar Cells. J. Mater. Chem. A 2013, 1, 8694-8709.

(8) Dang, M. T.; Hirsch, L.; Wantz, G. P3HT:PCBM, Best Seller in Polymer Photovoltaic Research. Adv. Mater. 2011, 23, 3597-3602.

(9) Vaynzof, Y.; Kabra, D.; Zhao, L.; Ho, P. K. H.; Wee, A. T.-S.; Friend, R. H. Improved Photoinduced Charge Carriers Separation in Organic-Inorganic Hybrid Photovoltaic Devices. Appl. Phys. Lett. 2010, 97, 033309.

(10) Park, S. H.; Roy, A.; Beaupre, S.; Cho, S.; Coates, N.; Moon, J. S.; Moses, D.; Leclerc, M.; Lee, K.; Heeger, A. J. Bulk Heterojunction Solar Cells with Internal Quantum Efficiency Approaching 100\%. Nat. Photonics 2009, 3, 297-302.

(11) Brédas, J.-L.; Norton, J. E.; Cornil, J.; Coropceanu, V. Molecular Understanding of Organic Solar Cells: The Challenges. Acc. Chem. Res. 2009, 42, 1691-1699.

(12) Clarke, T. M.; Durrant, J. R. Charge Photogeneration in Organic Solar Cells. Chem. Rev. 2010, 110, 6736-6767.

(13) Deibel, C.; Strobel, T.; Dyakonov, V. Role of the Charge Transfer State in Organic Donor-Acceptor Solar Cells. Adv. Mater. 2010, 22, 4097-4111.

(14) Grancini, G.; Maiuri, M.; Fazzi, D.; Petrozza, A.; Egelhaaf, H. J.; Brida, D.; Cerullo, G.; Lanzani, G. Hot Exciton Dissociation in Polymer Solar Cells. Nat. Mater. 2013, 12, 29-33.

(15) Bakulin, A. A.; Rao, A.; Pavelyev, V. G.; van Loosdrecht, P. H. M.; Pshenichnikov, M. S.; Niedzialek, D.; Cornil, J.; Beljonne, D.; Friend, R. H. The Role of Driving Energy and Delocalized States for Charge Separation in Organic Semiconductors. Science 2012, 335, $1340-1344$.

(16) Chen, K.; Barker, A. J.; Reish, M. E.; Gordon, K. C.; Hodgkiss, J. M. Broadband Ultrafast Photoluminescence Spectroscopy Resolves Charge Photogeneration via Delocalized Hot Excitons in Polymer:Fullerene Photovoltaic Blends. J. Am. Chem. Soc. 2013, 135, 1850218512.

(17) Tamura, H.; Burghardt, I. Ultrafast Charge Separation in Organic Photovoltaics Enhanced by Charge Delocalization and Vibronically Hot Exciton Dissociation. J. Am. Chem. Soc. 2013, 135, 16364-16367.

(18) Gélinas, S.; Rao, A.; Kumar, A.; Smith, S. L.; Chin, A. W.; Clark, J.; van der Poll, T. S.; Bazan, G. C.; Friend, R. H. Ultrafast Long-Range
Charge Separation in Organic Semiconductor Photovoltaic Diodes. Science 2014, 343, 512-516.

(19) Lee, J.; Vandewal, K.; Yost, S. R.; Bahlke, M. E.; Goris, L.; Baldo, M. A.; Manca, J. V.; Van Voorhis, T. Charge Transfer State Versus Hot Exciton Dissociation in Polymer-Fullerene Blended Solar Cells. J. Am. Chem. Soc. 2010, 132, 11878-11880.

(20) van der Hofstad, T. G. J.; Di Nuzzo, D.; van den Berg, M.; Janssen, R. A. J.; Meskers, S. C. J. Influence of Photon Excess Energy on Charge Carrier Dynamics in a Polymer-Fullerene Solar Cell. Adv. Energy Mater. 2012, 2, 1095-1099.

(21) Vandewal, K.; Albrecht, S.; Hoke, E. T.; Graham, K. R.; Widmer, J.; Douglas, J. D.; Schubert, M.; Mateker, W. R.; Bloking, J. T.; Burkhard, G. F.; et al. Efficient Charge Generation by Relaxed ChargeTransfer States at Organic Interfaces. Nat. Mater. 2014, 13, 63-68.

(22) Jailaubekov, A. E.; Willard, A. P.; Tritsch, J. R.; Chan, W.-L.; Sai, N.; Gearba, R.; Kaake, L. G.; Williams, K. J.; Leung, K.; Rossky, P. J.; et al. Hot Charge-Transfer Excitons Set the Time Limit for Charge Separation at Donor/Acceptor Interfaces in Organic Photovoltaics. Nat. Mater. 2013, 12, 66-73.

(23) Vaynzof, Y.; Bakulin, A. A.; Gelinas, S.; Friend, R. H. Direct Observation of Photoinduced Bound Charge-Pair States at an Organic-Inorganic Semiconductor Interface. Phys. Rev. Lett. 2012, $108,246605$.

(24) Wöll, C. The Chemistry and Physics of Zinc Oxide Surfaces. Prog. Surf. Sci. 2007, 82, 55-120.

(25) Adachi, S. Properties of Group-IV, III-V and II-VI Semiconductors; John Wiley \& Sons, Ltd: Chichester, U.K., 2005.

(26) Dag, S.; Wang, L.-W. Modeling of Nanoscale Morphology of Regioregular Poly(3-Hexylthiophene) on a Zno (1010) Surface. Nano Lett. 2008, 8, 4185-4190.

(27) Sai, N.; Leung, K.; Chelikowsky, J. R. Hybrid Density Functional Study of Oligothiophene/ZnO Interface for Photovoltaics. Phys. Rev. B 2011, 83, 121309.

(28) Noori, K.; Giustino, F. Ideal Energy-Level Alignment at the $\mathrm{ZnO} / \mathrm{P} 3 \mathrm{HT}$ Photovoltaic Interface. Adv. Funct. Mater. 2012, 22, 5089-5095.

(29) Brinkmann, M.; Rannou, P. Effect of Molecular Weight on the Structure and Morphology of Oriented Thin Films of Regioregular Poly(3-Hexylthiophene) Grown by Directional Epitaxial Solidification. Adv. Funct. Mater. 2007, 17, 101-108.

(30) Brinkmann, M.; Wittmann, J. C. P3HT-Structure-ExperimentalOrientation of Regioregular Poly(3-Hexylthiophene) by Directional Solidification: A Simple Method to Reveal the Semicrystalline Structure of a Conjugated Polymer. Adv. Mater. 2006, 18, 860-863.

(31) Oosterhout, S. D.; Koster, L. J. A.; van Bavel, S. S.; Loos, J.; Stenzel, O.; Thiedmann, R.; Schmidt, V.; Campo, B.; Cleij, T. J.; Lutzen, L.; Vanderzande, D.; Wienk, M. M.; Janssen, R. A. J.; et al. Controlling the Morphology and Efficiency of Hybrid Zno:Polythiophene Solar Cells via Side Chain Functionalization. Adv. Energy Mater. 2011, 1, 90-96.

(32) Falke, S. M.; Rozzi, C. A.; Brida, D.; Maiuri, M.; Amato, M.; Sommer, E.; De Sio, A.; Rubio, A.; Cerullo, G.; Molinari, E.; et al. Coherent Ultrafast Charge Transfer in an Organic Photovoltaic Blend. Science 2014, 344, 1001-1005.

(33) Li, Z.; Zhang, X.; Lu, G. Electron Structure and Dynamics at Poly(3-Hexylthiophene)/Fullerene Photovoltaic Heterojunctions. Appl. Phys. Lett. 2011, 98, 083303.

(34) Li, Z.; Zhang, X.; Lu, G. Dipole-Assisted Charge Separation in Organic-Inorganic Hybrid Photovoltaic Heterojunctions: Insight from First-Principles Simulations. J. Phys. Chem. C 2012, 116, 9845-9851.

(35) Dutton, G. J.; Robey, S. W. Distance Dependence of Exciton Dissociation at a Phthalocyanine- $-\mathrm{C}_{60}$ Interface. J. Phys. Chem. C 2013, 117, 25414-25423.

(36) Caruso, D.; Troisi, A. Long-Range Exciton Dissociation in Organic Solar Cells. Proc. Natl. Acad. Sci. U.S.A. 2012, 109, 1349813502. 
(37) Widmer, J.; Tietze, M.; Leo, K.; Riede, M. Open-Circuit Voltage and Effective Gap of Organic Solar Cells. Adv. Funct. Mater. 2013, 23, 5814-5821.

(38) Isaacs, E. B.; Sharifzadeh, S.; Ma, B.; Neaton, J. B. Relating Trends in First-Principles Electronic Structure and Open-Circuit Voltage in Organic Photovoltaics. J. Phys. Chem. Lett. 2011, 2, 25312537.

(39) Zhang, X.; Li, Z.; Lu, G. First-Principles Simulations of Exciton Diffusion in Organic Semiconductors. Phys. Rev. B 2011, 84, 235208.

(40) Akimov, A. V.; Neukirch, A. J.; Prezhdo, O. V. Theoretical Insights into Photoinduced Charge Transfer and Catalysis at Oxide Interfaces. Chem. Rev. 2013, 113, 4496-4565.

(41) Tully, J. C. Molecular Dynamics with Electronic Transitions. J. Chem. Phys. 1990, 93, 1061-1071.

(42) Duncan, W. R.; Craig, C. F.; Prezhdo, O. V. Time-Domain Ab Initio Study of Charge Relaxation and Recombination in DyeSensitized $\mathrm{TiO}_{2}$. J. Am. Chem. Soc. 2007, 129, 8528-8543.

(43) Fischer, S. A.; Habenicht, B. F.; Madrid, A. B.; Duncan, W. R.; Prezhdo, O. V. Regarding the Validity of the Time-Dependent KohnSham Approach for Electron-Nuclear Dynamics via Trajectory Surface Hopping. J. Chem. Phys. 2011, 134, 024102.

(44) Li, Z.; Zhang, X.; Woellner, C. F.; Lu, G. Understanding Molecular Structure Dependence of Exciton Diffusion in Conjugated Small Molecules. Appl. Phys. Lett. 2014, 104, 143303.

(45) Li, Z.; Zhang, X.; Lu, G. Exciton Diffusion in Disordered Small Molecules for Organic Photovoltaics: Insights from First-Principles Simulations. J. Phys.: Condens. Matter. 2014, 26, 185006.

(46) Mou, W.; Ohmura, S.; Shimojo, F.; Nakano, A. Molecular Control of Photoexcited Charge Transfer and Recombination at a Quaterthiophene/Zinc Oxide Interface. Appl. Phys. Lett. 2012, 100, 203306.

(47) Duncan, W. R; Stier, W. M.; Prezhdo, O. V. Ab Initio Nonadiabatic Molecular Dynamics of the Ultrafast Electron Injection across the Alizarin- $\mathrm{TiO}_{2}$ Interface. J. Am. Chem. Soc. 2005, 127, 7941-7951.

(48) Meng, S.; Ren, J.; Kaxiras, E. Natural Dyes Adsorbed on $\mathrm{TiO}_{2}$ Nanowire for Photovoltaic Applications: Enhanced Light Absorption and Ultrafast Electron Injection. Nano Lett. 2008, 8, 3266-3272.

(49) Meng, S.; Kaxiras, E. Electron and Hole Dynamics in DyeSensitized Solar Cells: Influencing Factors and Systematic Trends. Nano Lett. 2010, 10, 1238-1247.

(50) Neukirch, A. J.; Hyeon-Deuk, K.; Prezhdo, O. V. Time-Domain $\mathrm{Ab}$ Initio Modeling of Excitation Dynamics in Quantum Dots. Coord. Chem. Rev. 2014, 263-264, 161-181.

(51) Tretiak, S.; Igumenshchev, K.; Chernyak, V. Exciton Sizes of Conducting Polymers Predicted by Time-Dependent Density Functional Theory. Phys. Rev. B 2005, 71, 033201.

(52) Refaely-Abramson, S.; Sharifzadeh, S.; Govind, N.; Autschbach, J.; Neaton, J. B.; Baer, R.; Kronik, L. Quasiparticle Spectra from a Nonempirical Optimally Tuned Range-Separated Hybrid Density Functional. Phys. Rev. Lett. 2012, 109, 226405.

(53) Kroon, R.; Lenes, M.; Hummelen, J. C.; Blom, P. W. M.; de Boer, B. Small Bandgap Polymers for Organic Solar Cells (Polymer Material Development in the Last 5 Years). Polym. Rev. 2008, 48, $531-582$.

(54) Kresse, G.; Furthmüller, J. Efficient Iterative Schemes for $a b$ Initio Total-Energy Calculations Using a Plane-Wave Basis Set. Phys. Rev. B 1996, 54, 11169-11186.

(55) Blöchl, P. E. Projector Augmented-Wave Method. Phys. Rev. B 1994, 50, 17953-17979.

(56) Grimme, S. Semiempirical GGA-Type Density Functional Constructed with a Long-Range Dispersion Correction. J. Comput. Chem. 2006, 27, 1787-1799.

(57) Heyd, J.; Scuseria, G. E.; Ernzerhof, M. Erratum: "Hybrid Functionals Based on a Screened Coulomb Potential” [J. Chem. Phys. 118, 8207 (2003)]. J. Chem. Phys. 2006, 124, 219906. 